\title{
Structure and Elastic Properties of Networks Formed by Random Cross-Linking of Star Polymers ${ }^{\dagger}$
}

\author{
Jean Pierre QUeSLEL and James E. MARK \\ Department of Chemistry and the Polymer Research Center, The University of Cincinnati, \\ Cincinnati, Ohio 45221, U.S.A.
}

(Received September 30, 1985)

\begin{abstract}
The topology of networks formed by random cross-linking of star polymers having $A$ arms is investigated. It is shown that simple relationships exist between the molecular weight $M_{\mathrm{c}}$ between junctions, the number average molecular weight $M_{n}$ of the primary star arms, the cycle rank $\xi$, the total number density $\mu / V_{\mathrm{o}}$ of junctions, and the average junction functionality $\phi_{\mathrm{av}}$ for regular networks having no defects other than chain ends. The revised Flory-Erman theory treating entanglements as restrictions on junction fluctuations is used to predict moduli and swelling equilibrium properties of cis-polyisoprene networks prepared from 3-arm and 4-arm polymers of varying $M_{n}$.

KEY WORDS Elasticity / Random Networks / Star Polymers / Flory-

Erman Theory / Junction Fluctuations / Cycle Rank / cis-Polyisoprene /

Moduli / Swelling Equilibrium /
\end{abstract}

In a previous paper, ${ }^{1}$ the topology of networks formed by random cross-linking of linear polymers was studied. For regular networks having no imperfections other than chain ends, an expression relating $\xi$, the cycle rank or number of independent circuits in the network, ${ }^{2}$ the molecular weight $M_{\mathrm{c}}$ between cross-links, and the number average molecular weight $M_{n}$ of the primary linear chains was proposed. It was then shown ${ }^{3}$ that this relationship and the revised Flory-Erman elasticity theory of real networks ${ }^{4}$ can be used together to describe the elastic and swelling properties of statistical networks.

As many studies are now being focused on the rheological and viscoelastic properties of star-branched polymers, ${ }^{5-8}$ it is important to describe the structure of random networks formed from star polymers and to predict their elastic and swelling.properties in the equilibrium limit.

\section{THEORY}

According to Flory, ${ }^{2,9}$ a network structure can be characterized by the value of its cycle rank $\xi$, which subsumes all aspects of network imperfections. It is defined as the number of chains which have to be cut to reduce the network to an acyclic structure or tree, and is a universal measure of connectivity. ${ }^{10}$

Scanlan ${ }^{11}$ and Case $^{12}$ have defined an active junction as one joined by at least three paths to the gel (network), and an active chain as one terminated by an active junction at both ends. It was later shown that the difference between the numbers of active chains $v$ and junctions $\mu$ is the cycle rank $^{10,13}$

$$
\xi=v-\mu
$$

In the network model developed here, $A$-arm star primary chains $(A>2)$ are connected by tetrafunctional junctions in a random, non-

\footnotetext{
${ }^{\dagger}$ It is a pleasure to dedicate this paper to Dr. P. Thirion on the occasion of his retirement.
} 
degradative way. The distribution of arm molecular weights is narrow and characterized by its number-average value $M_{n}$. Moreover it is assumed that the mesh size $M_{\mathrm{c}}$ (molecular weight between cross-links) has a narrow distribution and also characterizes the dangling chains. These conditions are plausible and would be attained for polymers polymerized and branched in a controlled manner. The number of junctions which connect two or three dangling chains is probably relatively small in typical networks. In the following treatment, such junctions are therefore ignored and the final relationships are thus only valid in a first approximation. Another consequence of this assumption is that all the junctions are active.

Although there is no limitation on the $A$ value in this topological study, applications of molecular theories of rubberlike elasticity require that the networks have junctions which can be treated as points. ${ }^{14}$ Large values of $A$ are therefore not covered.

In the precursor liquid, the average molecular weight of the $A$-arm stars in $A M_{n}$, and the number density of chain ends is $\rho / M_{n}$. In the final network, the number density $\mu_{\mathrm{o}} / V_{\mathrm{o}}$ of $A$-functionality junctions provided by the star vertices is equal to the number density of initial stars, i.e., $\rho / A M_{n}$ :

$$
\mu_{\mathrm{o}} / V_{\mathrm{o}}=\rho / A M_{n}
$$

where $V_{\mathrm{o}}$ is the reference state volume and $\rho$ the polymer density.

Consider now two initial stars. As illustrated in Figure 1, the simplest way to connect them without any inactive junction is to use six tetrafunctional junctions, i.e., one junction per arm. It is easy to generalize this first-step network since $\rho / M_{n}$ tetrafunctional crosslinks are necessary to connect $\rho / A M_{n}$ stars per unit volume. The total number of junctions $\mu$ in the first-step network is therefore the sum of the initial star vertices $\mu_{\mathrm{o}}$ and these additional cross-links:

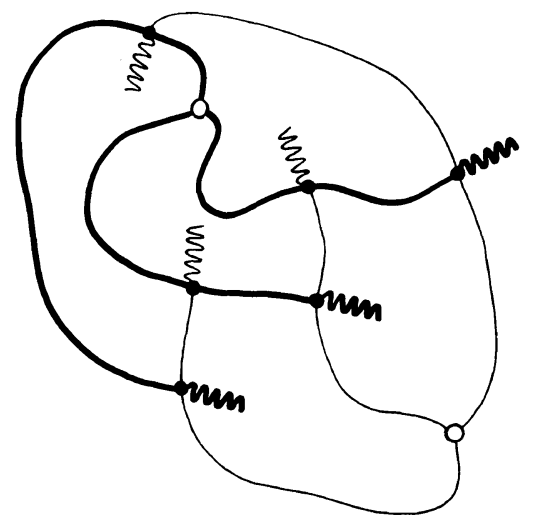

Figure 1. First-step network formed by connecting two 3-arm stars.

$$
\mu_{1} / V_{\mathrm{o}}=\mu_{\mathrm{o}} / V_{\mathrm{o}}+\rho / M_{n}
$$

At the end of this step, two tetrafunctional cross-links exist on each arm, creating two active chains per arm. Therefore the total number $v_{1}$ of active chains in the first-step network is twice the number of initial arms:

$$
v_{1} / V_{\mathrm{o}}=2 \rho / M_{n}
$$

When a tetrafunctional junction is then added, the number of active chains is increased by two. In reality, the cross-linking process is statistical and one can suppose that $M_{\mathrm{c}}$ at each step is regularly distributed within the network. A relationship between the final number of chains $v$ and junctions $\mu$ can be deduced to be

$$
v-v_{1}=2\left(\mu-\mu_{1}\right)
$$

The final density of active chains $v / V_{\mathrm{o}}$ is the difference between the total number density of chains of molecular weight $M_{\mathrm{c}}$ and the number density of chain ends: ${ }^{15}$

$$
v / V_{\mathrm{o}}=\rho / M_{\mathrm{c}}-\rho / M_{n}
$$

Note that in the usual chain-end correction for linear precursor chains $\rho / M_{n}$ in eq 6 is replaced by $2 \rho / M_{n}$.

Expressions of the total number density of active junctions and the cycle rank density are obtained by combination of eq $1-6$ : 


$$
\begin{aligned}
& \mu / V_{\mathrm{o}}=\left(\rho / 2 M_{\mathrm{c}}\right)\left[1-(A-2) M_{\mathrm{c}} / A M_{n}\right] \\
& \xi / V_{\mathrm{o}}=\left(\rho / 2 M_{\mathrm{c}}\right)\left(1-(A+2) M_{\mathrm{c}} / A M_{n}\right)
\end{aligned}
$$

In the case of 4-arm stars connected by tetrafunctional junctions, the overall functionality is four. However when $A$ differs from four, the resulting network is characterized by a number-average functionality $\phi_{\mathrm{av}}{ }^{16}$ which can be calculated using the equation

$$
\phi_{\mathrm{av}}=\left[A \mu_{\mathrm{o}}+4\left(\mu-\mu_{\mathrm{o}}\right)\right] / \mu
$$

Substitution of $\mu_{\mathrm{o}}$ and $\mu$ by their expressions given in eq 2 and 7, respectively, leads to

$$
\begin{gathered}
\phi_{\mathrm{av}}=4+2(A-4)\left[2-A+A M_{n} / M_{\mathrm{c}}\right]^{-1}(10) \\
\text { PREDICTIONS OF EQUILIBRIUM } \\
\text { ELASTICITY AND SWELLING } \\
\text { PROPERTIES }
\end{gathered}
$$

\section{PREDICTIONS OF EQUILIBRIUM ELASTICITY AND SWELLING PROPERTIES}

The revised Flory-Erman model of real networks has been successfully used to describe rubber elastic behavior. ${ }^{17-21}$ Consequently, it was chosen in this study to predict elastic and swelling properties of star precursor networks.

In this model, ${ }^{4,9}$ the restrictions of junction fluctuations due to neighboring chains are represented by domains of constraints. At small deformations, the stress is enhanced relative to that exhibited by a phantom network. At large strains, or high dilation, the effects of the restrictions on the fluctuations vanish and the relationship of stress to strain converges to that for the phantom case. The network behavior is described by two parameters, $\kappa$ and $\zeta$. The first, $\kappa$, measures the severity of entanglement constraints relative to those imposed by the phantom network connectivity, and $\zeta$ takes into account the nonaffine transformation of the domains of constraints with strain.

The Flory-Erman treatment leads to the following expression for the reduced force:

$$
\begin{aligned}
& {\left[f^{*}\right]_{\text {Flory }}} \\
& =\left(\xi / V_{\mathrm{o}}\right) R T\left\{1+2\left(\phi_{\mathrm{av}}-2\right)^{-1}\left[\alpha K\left(\alpha^{2} v_{2}^{-2 / 3}\right)\right.\right. \\
& \left.\left.\quad-\alpha^{-2} K\left(\alpha^{-1} v_{2}^{-2 / 3}\right)\right]\left(\alpha-\alpha^{-2}\right)^{-1}\right\}
\end{aligned}
$$

The function $K$ is defined in ref 4 (eq 37). It depends on $\kappa$ and $\zeta$, though not reproduced here. The quantity $v_{2}$ is the volume fraction of polymer in the swollen network, $R$ the gas constant, $T$ the absolute temperature, and $\alpha$ the extension ratio defined relative to the undeformed swollen state. The reduced force is defined by

$$
\left[f^{*}\right] \equiv f v_{2}^{1 / 3} / A_{\mathrm{d}}\left(\alpha-\alpha^{-2}\right)
$$

where $f$ is the measured force and $A_{\mathrm{d}}$ the cross-sectional area of the isotropic unswollen sample.

On the plausible grounds that the constraints on junctions are determined by the degree of interpenetration of chains and junctions in the network, the parameter $\kappa$ and the number of junctions $\mu$ in the volume $V_{\mathrm{o}}$ of the state of reference should be related ${ }^{17}$ according to

$$
\kappa=I\left\langle r^{2}\right\rangle_{\mathrm{o}}^{3 / 2}\left(\mu / V_{\mathrm{o}}\right)
$$

$I$ is the interpenetration parameter whose value appears to be $c a .0 .5,{ }^{17,18}$ and $\left\langle r^{2}\right\rangle_{\mathrm{o}}$ is the mean-square end-to-end length of the unperturbed network chain.

In the Flory-Erman model, the following equation describing network swelling equilibrium can be established:22

$$
\begin{aligned}
& \ln \left(1-v_{2 \mathrm{~m}}\right)+v_{2 \mathrm{~m}}+\chi v_{2 \mathrm{~m}}^{2} \\
& \quad=-\left(\xi / V_{\mathrm{o}}\right) V_{1} v_{2 \mathrm{~m}}^{1 / 3}\left[1+2\left(\phi_{\mathrm{av}}-2\right)^{-1} K\left(v_{2 \mathrm{~m}}^{-2 / 3}\right)\right]
\end{aligned}
$$

where $\chi$ is the solvent-polymer interaction parameter, $V_{1}$ the molar volume of solvent, and $v_{2 \mathrm{~m}}$ the volume fraction of polymer at swelling equilibrium.

Numerical applications presented in this section concern cis-polyisoprene 3- and 4-arm stars of varying $M_{n}$ cured with dicumyl per- 
Table I. Structure and elastic properties of $c i s$-polyisoprene networks formed from 3-arm and 4-arm star polymers of varying $M_{n}$

\begin{tabular}{|c|c|c|c|c|c|c|c|c|c|}
\hline $\begin{array}{c}\text { Network } \\
\text { designation }\end{array}$ & $A$ & $\frac{10^{-4} M_{n}}{\mathrm{~g} \mathrm{~mol}^{-1}}$ & $\frac{10^{5} \mu / V_{0}}{\mathrm{~mol} \mathrm{~cm}^{-3}}$ & $\frac{10^{-3} M_{\mathrm{c}}}{\mathrm{g} \mathrm{mol}^{-1}}$ & $\phi_{\mathrm{av}}$ & $\frac{\left[\mathrm{f}^{*}\right]_{\mathrm{ph}}^{\mathrm{a}}}{\mathrm{N} \mathrm{mm}^{-2}}$ & $\frac{\left[\mathrm{f}^{*}\right]_{\mathrm{aff}}^{\mathrm{a}}}{\mathrm{Nmm^{-2 }}}$ & $\kappa$ & $v_{2 \mathrm{~m}}$ \\
\hline 3IRO3 & 3 & 3 & 10.83 & 4.01 & 3.91 & 0.2183 & 0.4473 & 4.16 & 0.1883 \\
\hline 3IRO5 & 3 & 5 & 10.42 & 4.24 & 3.94 & 0.2283 & 0.4635 & 4.35 & 0.1925 \\
\hline 3IR10 & 3 & 10 & 10.12 & 4.43 & 3.97 & 0.2359 & 0.4753 & 4.50 & 0.1957 \\
\hline 3 IR 15 & 3 & 15 & 10.02 & 4.50 & 3.98 & 0.2384 & 0.4792 & 4.56 & 0.1967 \\
\hline 4IRO3 & 4 & 3 & 10.57 & 4.01 & 4 & 0.2246 & 0.4492 & 4.06 & 0.1900 \\
\hline 4IRO5 & 4 & 5 & 10.57 & 4.24 & 4 & 0.2321 & 0.4642 & 4.28 & 0.1935 \\
\hline 4IR 10 & 4 & 10 & 10.04 & 4.43 & 4 & 0.2377 & 0.4755 & 4.47 & 0.1961 \\
\hline 4 IR 15 & 4 & 15 & 9.97 & 4.50 & 4 & 0.2396 & 0.4793 & 4.54 & 0.1970 \\
\hline
\end{tabular}

aT $T=298 \mathrm{~K}$.

oxide (dicup). The number density of junctions can be calculated from knowledge of the initial amount $x$ of peroxide (weight percent in bulk). Full decomposition of peroxide and a $1: 1$ cross-linking efficiency are assumed since the purpose of these applications is to give realistic but qualitative values of the parameters describing network structure or governing elastic properties. The number density of junctions of the resulting network is the sum of the initial star vertex density and the density of C-C cross-links created by peroxide curing:

$$
\mu / V_{\mathrm{o}}=\rho / A M_{n}+\rho x /[270(100+x)]
$$

Typical values of $3 \mathrm{phr}$ for the initial amount of dicup, and 3, 5, 10, and $15 \times 10^{4} \mathrm{~g} \mathrm{~mol}^{-1}$ for $M_{n}$ were chosen. For cis-polyisoprene, $\left\langle r^{2}\right\rangle_{\mathrm{o}}$ and $M_{\mathrm{c}}$ are related through the equation: ${ }^{23-26}$

$$
\left\langle r^{2}\right\rangle_{\mathrm{o}}=3.8 \times 10^{7} M_{\mathrm{c}} / N_{\mathrm{A}}
$$

where $N_{\mathrm{A}}$ is Avogadro's number, and $M_{\mathrm{c}}$ and $\left\langle r^{2}\right\rangle_{\mathrm{o}}$ are expressed in $\mathrm{g} \mathrm{mol}^{-1}$ and $\mathrm{cm}^{2}$, respectively. Reasonable values of 0.0 and 0.5 were assigned $\zeta$ and $I$, respectively. The following relationships between the cycle rank and the phantom and affine moduli, $\left[f^{*}\right]_{\mathrm{ph}}$ and $\left[f^{*}\right]_{\text {aff }}$ respectively, have been established by Flory $^{2,9}$ and are used to calculate the phantom and affine moduli of the cis-polyisoprene networks.

$$
\begin{gathered}
{\left[f^{*}\right]_{\mathrm{ph}}=\left(\xi / V_{\mathrm{o}}\right) R T} \\
{\left[f^{*}\right]_{\mathrm{aff}}=\phi_{\mathrm{av}}\left(\phi_{\mathrm{av}}-2\right)^{-1}\left(\xi / V_{\mathrm{o}}\right) R T}
\end{gathered}
$$

Volume fractions of polymer $v_{2 \mathrm{~m}}$ at swelling equilibrium have been calculated by solving eq 14. Benzene was chosen as solvent ( $V_{1}=$ $89.33 \mathrm{~cm}^{3} \mathrm{~mol}^{-1}$ ). The required values of the interaction parameter $\chi$ of the system natural rubber + benzene were measured by Eichinger and Flory. ${ }^{27}$ The following polynomial function for $\chi$ versus $v_{2}$ was obtained by least-square analysis of the experimental data over the entire range of available $v_{2}:{ }^{3}$

$$
\chi=0.410+0.080 v_{2}+0.033 v_{2}^{2}
$$

Network designations and elasticity parameters calculated through eq 7, 8, 10, 13, and 15-18 are reported in Table I.

As the same number of tetrafunctional cross-links was introduced by the peroxide cure, the final differences in number density of junctions, $\mu / V_{\mathrm{o}}$, are due to the initial star vertices, the largest number of which is found in the network with smallest $A$ and $M_{n}$, i.e., 3IRO3. It is worth noting that $M_{\mathrm{c}}$ depends only on $M_{n}$, and not on $A$. This result is a consequence of the combination of eq 7 and 15:

$$
M_{\mathrm{c}}=\left\{2 x /[270(100+x)]+M_{n}^{-1}\right\}^{-1}
$$

At constant $M_{n}$, precursors contribute equal 


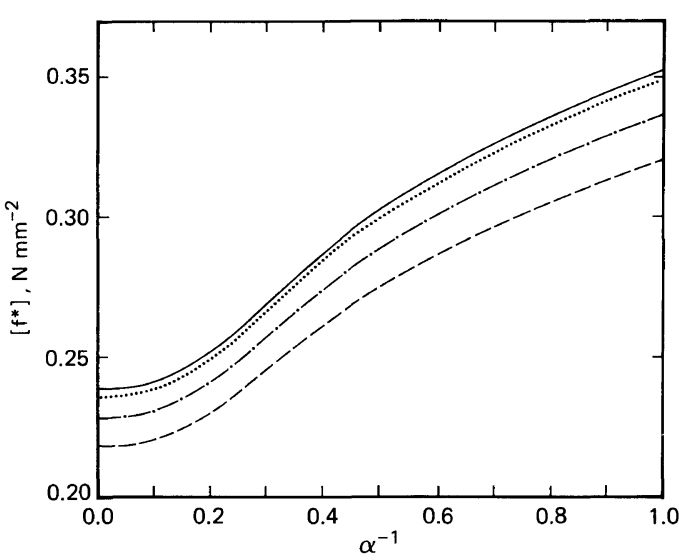

Figure 2. Stress-strain relationships in uniaxial extension for the four 3-arm star precursor networks characterized in Table I. Curves have been calculated from eq. 11 with the parameters given in Table I, and are for 3 IRO3

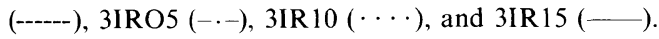

arm contour length whatever $A$ is. Therefore identical numbers of tetrafunctional crosslinks introduce the same number of segments of molecular weight $M_{\mathrm{c}}$. As usual, lower $M_{\mathrm{c}}$ corresponds to higher $\mu$. Increasing the 3-arm star molecular weight lowers the number density of trifunctional junctions relative to tetrafunctional ones introduced by the peroxide cure. A consequence is the increase in the average functionality with increase in $M_{n}$.

Stress-strain relationships in uniaxial extension for the 3-arm star precursor networks are represented in Figure 2. As a consequence of the increase of $\left[f^{*}\right]_{\mathrm{ph}}$ and $\kappa$ with $M_{n}$ (due to the decrease in number density of dangling chains), networks of higher $M_{n}$ exhibit superior moduli over the entire range of deformation (of course, this theory doesn't account for limited extensibility or possible straininduced crystallization).

Similar results are obtained for 4-arm star networks. The influence of the $A$ value is shown in Figure 3, where stress-strain relationships in uniaxial extension are presented for cis-polyisoprene networks formed from 3 and 4-arm stars of $M_{n}=3$ and $15 \times 10^{4} \mathrm{~g}$ $\mathrm{mol}^{-1}$, namely 3IRO3, 3IR 15, 4IRO3, and 4IR15. At constant $M_{n}$, 4-arm star precursor

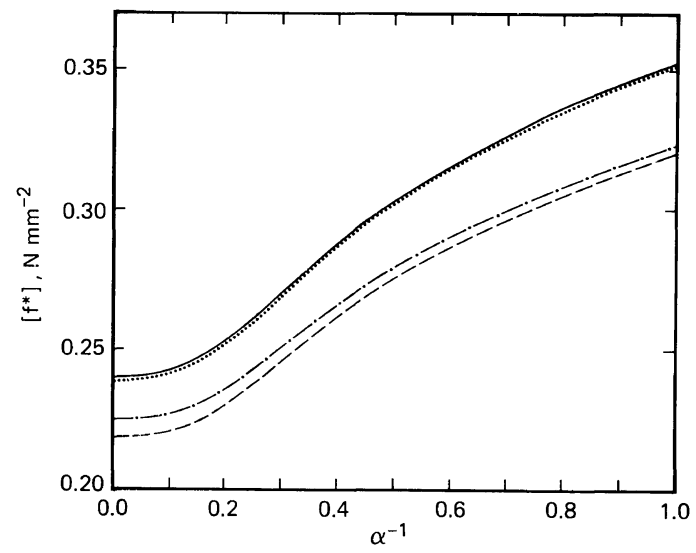

Figure 3. Stress-strain relationships in uniaxial extension for the cis-polyisoprene networks formed with 3and 4-arm stars having $M_{n}=3$ and $15 \times 10^{4} \mathrm{~g} \mathrm{~mol}^{-1}$ per arm. Curves have been calculated from eq 11 with the parameters given in Table I, and are for 3IRO3 (----),

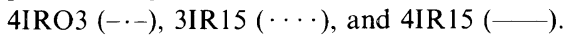

networks show higher moduli than those of 3arm star precursor networks. These differences are enhanced at low $M_{n}$ and in the large deformation range.

Values of $v_{2 \mathrm{~m}}$ for the eight networks are reported in the last column of Table I. They are in the same order as the moduli shown in Figures 2 and 3 , the highest value of $v_{2 \mathrm{~m}}$ corresponding as usual to the highest modulus.

\section{CONCLUSIONS}

Simple relationships have been established between parameters governing the structure and elasticity of star precursor polymers randomly cross-linked by tetrafunctional junctions and having no defects other than chain ends. These equations interrelate the initial star arm molecular weight and functionality, the final network cycle rank, the cross-link density, and the average functionality. Predictions of equilibrium elasticity and swelling properties of cis-polyisoprene networks were possible through the revised Flory-Erman elasticity theory for real networks. The results show an enhancement of 
modulus with increase in the initial star molecular weight and functionality.

Acknowledgements. It is a pleasure to acknowledge the financial support provided by the National Science Foundation through Grant DMR-8415082 (Polymers Program, Division of Materials Research). J. P. Queslel expresses his gratitude to the Michelin Tire Company for postdoctoral fellowship support.

\section{APPENDIX}

Star Precursor Networks with Chain End Molecular Weight $M_{\mathrm{o}}$ Different from $M_{\mathrm{c}}$

Equations $1-5$ still hold for this kind of network. If the chain ends have a molecular weight $M_{\mathrm{o}}$, there are $\left(M_{n}-M_{\mathrm{o}}\right) / M_{\mathrm{c}}$ active segments per primary arm, where $M_{\mathrm{c}}$ is now only the average molecular weight between cross-links. Since there are $\rho / M_{n}$ primary arms per unit volume, the total number density of active segments is

$$
v / V_{\mathrm{o}}=\left(\rho / M_{\mathrm{c}}\right)\left(1-M_{\mathrm{o}} / M_{n}\right)
$$

Combination of eq $1-5$ and 21 leads to the following expressions for the number densities of junctions, $\mu / V_{\mathrm{o}}$, and cycle rank density, $\xi / V_{0}$ :

$$
\begin{aligned}
& \mu / V_{\mathrm{o}}=\left(\rho / 2 M_{\mathrm{c}}\right)\left[1-\left(A M_{\mathrm{o}}-2 M_{\mathrm{c}}\right) / A M_{n}\right] \\
& \xi / V_{\mathrm{o}}=\left(\rho / 2 M_{\mathrm{c}}\right)\left[1-\left(A M_{\mathrm{o}}+2 M_{\mathrm{c}}\right) / A M_{n}\right]
\end{aligned}
$$

Equations 7 and 8 are recovered for $M_{\mathrm{o}}=$ $M_{\text {c. }}$.

\section{REFERENCES}

1. J. P. Queslel and J. E. Mark, J., Chem. Phys., 82, 3449 (1985).

2. P. J. Flory, Proc. R. Soc. London, Ser. A, 351, 351 (1976).

3. J. P. Queslel and J. E. Mark, paper to be published.

4. P. J. Flory and B. Erman, Macromolecules, 15, 800 (1982).

5. V. R. Raju, H. Rachapudy, and W. W. Graessley, J. Polym. Sci., Polym. Phys. Ed., 17, 1223 (1979).

6. W. W. Graessley, Adv. Polym. Sci., 47, 67 (1982).

7. W. W. Graessley, Macromolecules, 15, 1164 (1982).

8. W. W. Graessley and V. R. Raju, J. Polym. Sci., Polym. Symp., 71, 77 (1984).

9. P. J. Flory, Br. Polym. J., 17, 1 (1985).

10. P. J. Flory, Macromolecules, 15, 99 (1982).

11. J. Scanlan, J. Polym. Sci., 43, 501 (1960).

12. L. C. Case, J. Polym. Sci., 45, 397 (1960).

13. D. S. Pearson and W. W. Graessley, Macromolecules, 11, 528 (1978).

14. P. J. Flory and B. Erman, Macromolecules, 16, 595 (1983).

15. P. J. Flory, Chem. Rev., 35, 51 (1944).

16. J. P. Queslel and J. E. Mark, Polym. Bull., 12, 311 (1984).

17. B. Erman and P. J. Flory, Macromolecules, 15, 806 (1982).

18. B. Erman and P. J. Flory, Macromolecules, 16, 1607 (1983).

19. H. Pak and P. J. Flory, J. Polym. Sci., Polym. Phys. Ed., 17, 1845 (1979).

20. B. Erman, J. Polym. Sci., Polym. Phys. Ed., 19, 829 (1981).

21. B. Erman, J. Polym. Sci., Polym. Phys. Ed., 21, 893 (1983).

22. J. P. Queslel and J. E. Mark, Polym. Bull., 10, 119 (1983).

23. Y. Abe and P. J. Flory, Macromolecules, 4, 219, 230 (1971).

24. N. Hadjichristidis, X. Zhongde, and L. J. Fetters, J. Polym. Sci., Polym. Phys. Ed., 20, 743 (1982).

25. F. J. Ansorena, L. M. Revuelta, G. M. Guzmán, and J. J. Irvin, Eur. Polym. J., 18, 19 (1982).

26. J. Mays, N. Hadjichristidis, and L. J. Fetters, Macromolecules, 17, 2723 (1984).

27. B. E. Eichinger and P. J. Flory, Trans. Faraday Soc., 64, 2035 (1968). 